\title{
The Development of Evaluation Model for Internal Quality Assurance System of Dramatic Arts College of Bunditpattanasilpa Institute
}

\author{
Kittisak Sinthukhot ${ }^{1}$, Yannapat Srihamongkol ${ }^{2}$, Nuchwana Luanganggoon ${ }^{3} \&$ Paisan Suwannoi $^{3}$ \\ 1 Ph.D. Major Educational Research and Evaluation, Faculty of Education, Mahasarakham University, \\ Mahasarakham, Thailand \\ ${ }^{2}$ Advisor, Faculty of Education, Mahasarakham University, Mahasarakham, Thailand \\ ${ }^{3}$ Advisor, Faculty of Education, Khon Kaen University, Khon Kaen, Thailand \\ Correspondence: Kittisak Sinthukhot, Kalasin Colllege of Dramatic Arts, Kalasin, Thailand. E-mail: \\ sin_kitti@hotmail.com
}

Received: September 24, 2013 Accepted: October 11, 2013 Online Published: October 15, 2013

doi:10.5539/ies.v6n11p69 URL: http://dx.doi.org/10.5539/ies.v6n11p69

\begin{abstract}
The research purpose was to develop an evaluation model for the internal quality assurance system of the dramatic arts College of Bunditpattanasilpa Institute. The Research and Development method was used as research methodology which was divided into three phases; developing the model and its guideline, trying out the actual implementation of the developed model, and assessing and improving the model. Data collection processes started with interviewing experts in internal quality assurance system. Then the collected data was analyzed and synthesized to form a tentative model. The researcher spent eight months on trying out this tentative model. The four areas; Utility, Feasibility, Propriety, and Accuracy were used to evaluate and revise the model. Percentage, mean, and standard deviation were used in data analysis. The tentative model consisted of three steps; preparation, evaluation procedure, and reporting. This model was tried out at two institutes in Kalasin and Roi-Et Provinces. The evaluation result as a whole on Utility, Feasibility, Propriety, and Accuracy of the model by eighty users was at the "Highest" level (Mean = 4.51). The utility was at the "Highest" mean of 4.60. In conclusion, this evaluation model for internal quality assurance system could be used successfully at the dramatic arts College of Bunditpattanasilpa Institute.
\end{abstract}

Keywords: development, evaluation model, internal quality assurance system

\section{Introduction}

Education quality is necessary and important. It is a key goal of education management. Quality and mechanism for continuous improvement and upgrade of standard are needed in education management. Educational institutes publicize their performances among parents and communities in order to assure them that the co-built missions and education quality are met. According to the Constitution of the Kingdom of Thailand B.E. 2550 (2007) (The Constitution of the Kingdom of Thailand, 2007) and Chapter 6 of the National Education Act B.E. 2542 (1999) and Amendments (the Second National Education Act B.E.2545 (2002)), educational standards and quality assurance require quality assurance systems as stated in the following sections:

Section 47 There shall be a system of educational quality assurance to ensure improvement of educational quality and standards at all levels. Such a system shall be comprised of both internal and external quality assurance. The system, criteria, and methods for quality assurance shall be as stipulated in the ministerial regulations.

Section 48 Parent organizations with jurisdiction over educational institutions and the institutions themselves shall establish a quality assurance system in the institutions. Internal quality assurance shall be regarded as part of educational administration which must be a continuous process. This requires preparation of annual reports to be submitted to parent organizations, agencies concerned and made available to the public for purposes of improving the educational quality and standards and providing the basis for external quality assurance.

Section 49 All educational institutions shall receive external quality evaluation at least once every five years.

As aforementioned, educational quality assurance is a regular activity in the administrative management of educational institutes for continuous student quality improvement. It ensures educational quality for those who 
receive educational services. It also maintains quality improvement of students who will create future prosperity of societies and nations. Another important factor that can shape the future of a country is globalization. It nowadays is bringing about the advancement of information technology and rapid changes. It is essential that every country learn to catch up the emerging changes. They must be ready for the changes and challenges toward the quality of their citizens. Hence, Bunditpattanasilpa Institute, as a government educational institute of Thailand, has to make an adjustment and an improvement of educational service quality for the students of all its 12 dramatic arts College.

The dramatic arts College of Bunditpattanasilpa Institute provide education of arts and cultures, has excellence in producing graduates to be professional artists, academics, art teachers, researchers for national and local levels, and conserves and manifests national identity in the society. There are 12 dramatic arts College throughout the country. They perform an evaluation of both internal and external quality assurance of two levels; basic education (grades 7-12) and tertiary education. Bunditpattanasilpa Institute sets its basic education standards based on Office of the Basic Education Commission and Office for National Education Standards and Quality Assessment (public organization) whereas its tertiary education standards are based on Office of the Higher Education Commission.

The internal quality assurance system of the dramatic arts College has so far been developed under the announcements of Bunditpattanasilpa Institute. The system comprises three important elements for operation; quality control, quality check, and quality evaluation. The system emphasizes on input quality improvement, process, and output. The system itself leads to the uniform and continuous quality improvement of the performance of Bunditpattanasilpa Institute. It is also part of the institute's education administration system and process. Bunditpattanasilpa Institute assigns its College to develop quality standards and systems which aim at collaboration from all the staff levels; administration, operation, and individual person. Such collaboration pushes forward quality assurance systems in producing quality graduates with knowledge of arts and cultures into the national and global stages.

However, there has been a series of problems in the internal quality evaluation based on the quality assurance of the College of Bunditpattanasilpa Institute. According to the overall results from the internal basic education quality evaluation in the academic years 2007 and 2008, the evaluation committee indicated weaknesses that required improvement such as lack of a follow-up system, a filing system, raising awareness, and building understanding of evaluation standards and administrative management systems. Due to this fact, it is necessary to clearly promote and support internal quality assurance systems. It is also important to regularly follow up and evaluate mechanisms, which are related to all the standards of quality assurance. In addition, for internal tertiary education quality evaluation, the staff of all levels are required to raise awareness of the importance of quality assurance. A list of responsible staff with a system of quality check must be clearly set.

Based on the significance and problems in internal quality evaluation mentioned above, there was a need in developing an evaluation model for internal quality assurance system of the dramatic arts College, Bunditpattanasilpa Institute. To develop this evaluation model, the concepts and theories of system decision-oriented evaluation were incorporated. Some collaboration-oriented evaluation techniques such as utilization-focused approach, empowerment evaluation, and collaboratory evaluation were also employed. The utilization-focused approach aims at benefits that evaluated organizations will gain. The process of utilization-focused approach requires collaboration and negotiation between evaluators and evaluation target groups. The evaluation target groups are selected by evaluated organizations. The empowerment evaluation (EE) is designed to help drive improvement and determine a direction for self-performance. This type of evaluation applies both quantitative and qualitative methods. It is effective in empowering individuals, organizations, and communities. The empowerment evaluation is usually run in form of a project with these elements; training, facilitation, advocacy, illumination, and liberation. The collaboratory evaluation is an evaluative type which gathers all groups of stakeholders to take part in evaluation. It has a high degree of collaboration. Both evaluators and stakeholders possess equal right in making a decision of evaluation techniques. An important activity of collaboratory evaluation is making a discussion among evaluation team members until they share the same understanding. These collaboration-oriented evaluation techniques are appropriate for internal quality assurance systems of educational institutes. The development of an evaluation model in this research focused on the identity of dramatic arts College, Bunditpattanasilpa Institute, in that they provide specific science, produce graduates with knowledge of arts and cultures, research and creations, give academic services to the society, and preserve arts and cultures. The developed evaluation model was regarded as a factor that helped the internal quality assurance of the dramatic arts College achieve its goals. 


\subsection{Research Question}

What was an appropriate evaluation model for the internal quality assurance system of the dramatic arts College, Bunditpattanasilpa Institute?

\subsection{Research Purpose}

The purpose of this research was to develop an evaluation model for the internal quality assurance system of the dramatic arts college of Bunditpattanasilpa Institute. The aims of the development of an evaluation model were;

1) To develop an evaluation model and guideline for the internal quality assurance system,

2) To try out the actual implementation of the developed evaluation model,

3) To assess and improve the developed evaluation model.

\subsection{Research Scope}

1.3.1 The Evaluation Model was Developed for the Internal Quality Assurance System of the Dramatic Arts College of Bunditpattanasilpa Institute

An analysis and synthesis was done to models, indicators, and criteria of the evaluation of the internal quality assurance system, which covered long-term education management with basic and tertiary (bachelor's degrees) education programs. The analysis and synthesis covered the 12 dramatic arts College including 1) The College of Dramatic Arts, 2) Chiang Mai College of Dramatic Arts, 3) Angtong College of Dramatic Arts, 4) Sukhothai College of Dramatic Arts, 5) Lopburi College of Dramatic Arts, 6) Chanthaburi College of Dramatic Arts, 7) Suphanburi College of Dramatic Arts, 8) Nakhon Si Thammarat College of Dramatic Arts, 9) Phatthalung College of Dramatic Arts, 10) Nakhon Ratchasima College of Dramatic Arts, 11) Roi-Et College of Dramatic Arts, and 12) Kalasin College of Dramatic Arts. The researcher studied the 12 College' SARs, local and foreign research reports which were related to education quality assurance, and interviews ofeducation management-related individuals. The analysis of concepts for preparing an evaluation model for internal quality assurance systems of educational institutes was based on four sets of educational evaluation standards; utility standards, feasibility standards, propriety standards, and accuracy standards (Stufflebeam \& Shinkfield, 1990).

1.3.2 The Sources of Information for Developing an Evaluation Model for the Internal Quality Assurance System of the Dramatic Arts College of Bunditpattanasilpa Institute were as Follows

\subsubsection{The Analysis and Synthesis of Models, Indicators, and Criteria}

The analysis and synthesis of models, indicators, and criteria made use of the following two sources of information.

1.3.2.1.1 The Information of Theories, Concepts, Related Research as Well as Indicators and Standards was Divided into Two Parts

1) Basic education standards which were relevant with the standards of Bunditpattanasilpa Institute, Office of the Basic Education Commission, and external basic education quality evaluation.

2) Higher education standards which were relevant with the standards of Bunditpattanasilpa Institute, Office of the Higher Education Commission, external higher education quality evaluation, and factors in the administration systems of the dramatic arts College of Bunditpattanasilpa Institute.

1.3.2.1.2 The Information from the Interviews with Five Individuals Who were Administrators or Officers Responsible for Quality Assurance of Bunditpattanasilpa Institute

The information concerned the state, problems, and obstacles in internal quality assurance and a needed evaluation model for the internal quality assurance system.

\subsubsection{The Development of an Evaluation Model and its Guideline}

An analysis of ten experts' consensus on the appropriateness and feasibility in collecting empirical data of models and indicators was done. This group of experts consisted of experts in the education administration management of the dramatic arts College of Bunditpattanasilpa Institute and experts in quality evaluation.

\subsubsection{The Tryout}

The data collection for the tryout was done in the evaluation process of the internal quality assurance system. The individuals related to the evaluation process included administrators, teaching staff, and students of the dramatic arts College of Bunditpattanasilpa Institute. Two dramatic arts College, Roi-Et College of Dramatic Arts and Kalasin College of Dramatic Arts, were purposively sampled to do a field study. A field study could be 
done at these two College due to the reason that the developed model of the research incorporated both basic and higher education standards. The couple of standard sets were found at the two College as they provided basic education (grades 7-12) and higher education (bachelor's degrees). For higher education, students are enrolled in the Faculty of Music and Drama and the Faculty of Art.

\subsubsection{The Assessment of the Developed Evaluation Model}

A questionnaire was administered to examine opinions toward the developed evaluation model. The informants were the users of the developed evaluation model which was tried out in the two field study College.

\section{Method}

The method of this research derived from the literature study of the researcher. The study included concepts of evaluation model development and systematic evaluation, evaluation theories and types, and criteria theories. The data from this prior study was analyzed and synthesized to form an evaluation approach. The elements of the approach included evaluation goals, evaluation targets, indicators and criteria, evaluation methods, and evaluators' qualifications. The research was conducted with the principles of research and development. The research and development methodology was consistent with the development process of the evaluation model (the research product) as it required a prior study, a development, a tryout of implementation, and an assessment. The completeness of the process could lead to the efficiency of the developed model. The conduction of the research was divided into three phases as follows:

Phase 1 The development of the evaluation model for the internal quality assurance system of the dramatic arts College of Bunditpattanasilpa Institute.

1. Analyzed documents, research, concepts, and theories concerning education quality evaluation models, indicators, and criteria of the dramatic arts College of Bunditpattanasilpa Institute.

2. Interviewed experts to gain information of the state, problems, and obstacles in the evaluation of the internal quality assurance and a needed evaluation model for the internal quality assurance system.

3. Synthesized the results from the analysis of the above information to design a tentative evaluation model.

4. Studied the appropriateness and feasibility in collecting empirical data of modelsand indicators through an expert-based seminar.

5. Developed an evaluation model and its guideline.

6. Expert-checked the appropriateness of the evaluation model for further improvement.

Phase 2 The tryout of the implementation of the evaluation model at the two field study College, Roi-Et College of Dramatic Arts and Kalasin College of Dramatic Arts

Phase 3 The assessment and improvement of the developed evaluation model for the internal quality assurance system of the dramatic arts College of Bunditpattanasilpa Institute.

The actual users of the developed evaluation model assessed its four sets of standards; utility standards, feasibility standards, propriety standards, and accuracy standards.

\section{Data Analysis}

The data collected in the three phases was analyzed as follows:

1. The data from the documents and the interviews was analyzed to develop an evaluation model for the internal quality assurance system of the dramatic arts College of Bunditpattanasilpa Institute.

2. The data from the observations and the interview was analyzed and presented with description.

The data from the evaluation was analyzed with the validity of the evaluation model used at the two field study College.

3. The data from the opinion questionnaire was analyzed through mean and standard deviation.

\section{Results}

The results of the research are presented in three sections according to the research purposes.

Section 1 The results of the development and the use of the evaluation model for the internal quality assurance system of the dramatic arts College of Bunditpattanasilpa Institute

The internal quality assurance system for improvement of education quality and standards of the dramatic arts College of Bunditpattanasilpa Institute included these elements; 1) internal quality evaluation, 2) education 
quality follow-up and check, and 3) education quality improvement. The process of the internal quality assurance system of the dramatic arts College of Bunditpattanasilpa Institute was consisted of three steps.

Step 1 Preparation for Evaluation The preparation was consisted of appointing an evaluation committee of the internal quality assurance, meeting of the evaluation team, distributing an evaluation handbook, and making an evaluation schedule.

Step 2 Evaluation The evaluation was consisted of collecting, analyzing, interpreting, and summarizing the data and results. The results of the actual indicators, factors, and sections were compared to the evaluation criteria. The evaluation criteria had five rating scales (1.00-5.00) for each evaluated target aspect. A single aspect was regarded as "Pass" with a "High" level when it received a minimum average of 3.51 .

The operation of the evaluation

1. Specified education standards of the College.

2. Made an education management development plan which focused on the education standards of the College.

3. Managed administration and information system

4. Followed the education management development plan

5. Managed follow-up and check of education quality

6. Provided an internal quality evaluation based on the education standards

7. Made an annual report of the internal quality evaluation

8. Promoted continuous education quality improvement

Step 3 Overall report of plans and projects The overall report presented the performance of the College including indicators, factors, and sections in form of tables and descriptions. This report was presented to related groups of individuals.

Section 2 The results of the tryout of the evaluation model for the internal quality assurance system of the dramatic arts College of Bunditpattanasilpa Institute.

The tryout of the developed evaluation model was performed at the two field study College. The results of the tryout are presented below.

\section{Roi-Et College of Dramatic Arts}

The results of the internal quality assurance system evaluated by the developed model revealed that the operation appropriateness was at the "High" level with an average of 4.49. Also, the results of the evaluations of the internal quality assurance systems in the other dramatic arts College accorded with the suggestions and the evaluation of Roi-Et College of Dramatic Arts of the academic year 2011 done on September $3^{\text {rd }}-4^{\text {th }}$, 2012. The results also showed that the average of 15 basic education standards was 5.00 interpreted as "Very Good" meanwhile that of 9 higher education standards was 3.62 as "Good."

\section{Kalasin College of Dramatic Arts}

The results of the internal quality assurance system evaluated by the developed model unveiled that the operation appropriateness was at the "High" level with an average of 3.99. The results of the evaluations of the internal quality assurance systems in the other dramatic arts College accorded with the suggestions and the evaluation of Kalasin College of Dramatic Arts of the academic year 2011 done on August $30^{\text {th }}-31^{\text {st }}, 2012$. It was also found that the average of the 15 basic education standards was 4.00 interpreted as "Good" whereas that of the 9 higher education standards was 2.87 as "Moderate."

Section 3 The results of the assessment of the developed evaluation model for the internal quality assurance system of the dramatic arts College of Bunditpattanasilpa Institute.

The participants in the assessment were 80 actual users of the developed evaluation model. They were administration and teaching staff of the two field study College. These assessors evaluated the four sets of standards; utility standards, feasibility standards, propriety standards, and accuracy standards. It was found that the overall standards were at the "High" level with an average of 4.51 . The utility standards were rated at the "Highest" level with an average of 4.60. The users also expressed problems and obstacles in using the developed evaluation model. These suggestions were incorporated into the further improvement of the developed model. Thus, more details resulting from the improvement were added to the guideline of the model. The more details included the identity of the College, instructions, criteria, explanations, and qualifications of assessors. The major focus was the identity of all the dramatic arts College. 


\section{Discussion}

There are three issues for the discussions; 1) the development of the evaluation model and 2) the instruments for the data collection, analysis, and summary.

\subsection{The Development of the Evaluation Model}

The evaluation model of this research was developed through expert criticism, suggestions, and tryout. The actual use of the developed model was done at the two field study College. The aim of the actual use was to study the results of the model. The development of the evaluation model in this research accorded with that of Tirawat Yemsang (2007) in that his model was developed through a preliminary study of the state of knowledge management. The tentative model was then tried out and improved to be a complete model. The present research was also in accordance with that of Chanoknart Cheunchey (2007). In that research, the researcher developed a model from a prior study of related literature. After that, she analyzed and synthesized the elements of gathered models. To improve the model, she incorporated expert opnions and measured its efficiency. In addition, the present research accorded with the following studies. Phiyapong Saisophon (2007) completed his model by validating, trying out, testing its efficiency, and further improving the model. Sathaporn Sathugarn (2007) developed his model through determining the efficiency and comparing the effectiveness before and after the model was used. Ascharavadee Sawatsuk (2006) developed a model by testing its quality through participatory research and invented an evaluation model by trying out and checking its system.

Based on the results of the actual use of the developed model at the two field study College, the averages of the standards were at the "High" and "Highest" levels. The elements of evaluation included 1) specifying education standards of the College, 2) making an education management development plan which focused on the education standards of the College, 3) managing administration and information system, 4) following the education management development plan, 5) managing follow-up and check of education quality, 6) providing an internal quality evaluation based on the education standards, 7) making an annual report of the internal quality evaluation, 8 ) promoting continuous education quality improvement.

The results of the evaluation of the internal quality assurance system in this research showed that the system passed the evaluation preset criteria. This finding accorded with 1) the study of the performance of education quality assurance of primary schools under Nakhon Si Thammarat Primary Educational Service Area Office 3 by Kusol Thepsiri (2005), 2) the study of the performance of education quality assurance of private schools under Nakhon Si Thammarat Primary Educational Service Area Office 2 by Chalermchai Hankla (2002), and 3) the evaluation of the internal quality assurance systems of basic education institutes under Nakhon Si Thammarat Primary Educational Service Area Office 2. The similar findings between this research and the previous studies resulted from the following factors.

1) The specification of education standards of the College enabled all the internal staff members to adjust to their specific responsibilities.

2) The education management development plan focused on the education standards of the College. Making a plan is important as it is the first out of the four steps to follow and to achieve the goals effectively.

3) The management of the administration and information system helped promote, support, and facilitate the internal quality assurance.

4) Almost all the elements of the education management development plan were followed.

5) Managing follow-up and check of education quality indicated an upgrade of checking and reviewing education quality in the report of evaluation. Checking and reviewing education quality is an important mechanism to activate improvement and feedbacks.

6) The provision of an internal quality evaluation based on the education standards resulted in greater understanding among administrators and teachers. As a consequence, they could effectively perform an internal quality evaluation.

7) The annual report of the internal quality evaluation is a good factor. It, however, was not utilized and publicized concretely. This might be because the staff lacked report writing skills or the instructions in the report were not clearly stated. Thus, a procedure and plan for publicizing the annual report should be made. This will not only raise awareness of educational quality assurance, but also call for collaboration from parents and communities.

8) The promotion of continuous education quality improvement was a necessary factor to develop and assess the performance of education quality assurance systems. 
As part of the development of the evaluation model for the internal quality assurance system in

this research, qualifications of experts who validated, criticized, and suggested improvement of the developed model were specified. Only experts in education quality assurance and measurement and evaluation in education were selected. The main reason was that the further improvement of the developed model after its tryout had to suit the context of dramatic arts College of Bunditpattanasilpa Institute. The completed model was to be applicable. After its tryout, the developed model was improved based on the analysis and synthesis of the data collected through documents and interviews in the research process. The improvement of the developed model was made systematically. This systematic improvement had a positive effect on the appropriateness and completeness of the model.

\subsection{The Instruments for the Data Collection, Analysis, and Summary}

To follow the whole evaluation process, the researcher thoroughly reviewed the steps in the designed evaluation model. A study of concepts and theories concerning constructing and developing evaluation instruments was done. The concepts of the evaluation instruments in this research were based on (Arlen Gulicson , 2008) who confirmed the four sets of evaluation standards; utility standards, feasibility standards, propriety standards, and accuracy standards. They were also in accordance with Davies's concepts of evaluation indicators and criteria (Phadungchai Pupat,1972). The evaluation criteria in this research were classified as scientific criteria, performance criteria, and value criteria. The evaluation approach of the model was consistent with the six approaches (Worthen and Sander, 1973).

The characteristics of the evaluation instruments in this research were consistent with the concepts of (Popham, 1999) in that such instruments had to have validity, reliability, discrimination, objectivity, difficulty, exemplary, searching, fairness, and efficiency. The construction process of the evaluation instruments was in accord with the concepts. There were eight stages in the construction process; 1) set measurement steps, 2) define target objects for measurement, 3) select types of measurement instruments, 4) construct measurement instruments, 5) try out constructed instruments, 6) test the quality of constructed instruments, 7) improve constructed instruments, and 8) make a handbook for completed instruments. The handbook of the evaluation model in this research was developed under the above concepts and theories. The data collection, analysis, interpretation, and summary of the evaluation results were in accordance with the concepts and theories on principles of evaluation data analysis

It was discovered that most of the results of the internal quality assurance system evaluated by the developed model were at the "High" level. This accorded with the results of the internal quality assurance of Bunditpattanasilpa Institute. It can be summarized that this was because most of the dramatic arts College did not totally perform the Plan-Do-Check-Act (PDCA) circle. They also lacked follow-up and check-up systems as well as awareness of importance of internal quality assurance.

\section{References}

Ascharavadee Sawatsuk. (2006). A Development of Authentic Assessment Model for Thai Language Subjects in Mathayomsuksa1. Dissertation of Eductional Measurement. Srinakarinwirot University: Thailand.

Chalermchai Hankla. (2002). The Development of Internal Quality Audit System of Rajabhat Institutes. Dissertation of Educational Administration. Chulalongkorn University: Thailand.

Chanoknart Cheunchey. (2007). A Model for Continuing Education Management in Private Higher Education Institutions. Dissertation of Adult Education. Srinakarinwirot University: Thailand.

Gullickson, Arlen. (2008). The Student Evaluation Students. National Conference on Evaluation Naresuan University, 5(7), 32.

Kemmis, S., \& McTaggart. (1988). The action research planner. Victoria: Deakin University.

Kusol Thepsiri. (2005). A Study of the Performance of Educational Quality Assurance in the Primary-level Schools under the Office of NaKhon si Thammarat Edcational Service Area 3. Dissertation of Educational Administration. Nakhon si Thammarat Rajabhat University: Thailand.

Phadungchai Pupat. (1972). A Comparison of Reliability and Discrimination Power of an English Test Scored by the Conventional. Dissertation of Eductional Measurement. Khon Kaen University: Thailand.

Phiyapong Saisophon. (2007). Participatory Learning Model Development of Life Skills Development Subject 1 Under Basic Education Curriculum B.E.2544 for Enhancing Life Skills of Nonformal Education Students in Central Correctional Institution. Dissertation of Adult Education. Srinakarinwirot University: Thailand.

Popham, W. James. (1999). Modern Education Measurement. Englewood Cliffs, New Jersey: Pretice-Hall. 
Sathaporn Sathugarn. (2007). The Development of an Appropriate Online Instruction Model for Deaf Students at Higher Education Level. Dissertation of Educational Technology. Srinakarinwirot University: Thailand.

Scriven, M. (1969). The Methodology of Evaluation. AERA Monograph Series in Curriculum Evaluation. No. 1. Chicago: Rand McNally.

Stufflebeam, D. L., \& Shinkfield A. J. (1990). Evaluation Theory, Models and Applications. San Francisco: Jossey-Bass, 2007.

Stufflebeam, D. L., \& others. (1971). Educational Evaluation and Decision Making. Itasca, Illinois: Peacock Publishing.

Stufflebeam, D. L. (1981). Meta Evaluation: Concepts, Standard, and Users. Education Evaluation Methodology: The State of the Art. The Johns Hopkins University Press.

Tirawar Yeamsang. (2007). Developing a Khowledge Management Model of the Office of Mahasarakham Educational Service Area Zone 1. Dissertation of Educational Adminstation and Development. Mahasarakham University: Thailand.

Worthen, B. R., \& Sanders, J. R. (1973). Educational Evaluation: Theory and Practice. Ohio: Charles A. Jones Publishing Company.

Worthen, B. R., \& Sanders, Jody L. Fitzpatrick. (1997). Program Evaluation Alternative Approaches and Practical Guidelines. New York: Longman.

\section{Copyrights}

Copyright for this article is retained by the author(s), with first publication rights granted to the journal.

This is an open-access article distributed under the terms and conditions of the Creative Commons Attribution license (http://creativecommons.org/licenses/by/3.0/). 